\title{
Thermography on moving samples: recent developments for quantitative evaluation
}

\author{
by A. Mendioroz, A. Salazar
}

\author{
*Universidad del País Vasco UPV/EHU, Departamento de Física Aplicada I, Escuela de Ingeniería de Bilbao, \\ Plaza Ingeniero Torres Quevedo 1, 48013 Bilbao, Spain, arantza.mendioroz@ehu.eus, agustin.salazar@ehu.es
}

\begin{abstract}
We propose a methodology to evaluate the thermal diffusivity and to size the thermal resistance of vertical cracks in samples that move at constant velocity, as they are illuminated by a continuous wave (cw) laser spot. This configuration is aimed at implementing in-line testing of industrial products that move at constant velocity, without stopping the production chain. First, we calculate the evolution of the surface temperature distribution of sound samples, and we propose a method to measure the in-plane thermal diffusivity of the material in the directions parallel and perpendicular to the motion. Second, we present calculations of the surface temperature distribution when the material contains an infinite vertical crack and we propose a methodology to size the width of the crack. In both cases, we present experimental data obtained on reference and calibrated samples that confirm the validity of the methods.
\end{abstract}

\section{Introduction}

Laser spot thermography is a well established technique to measure the thermal diffusivity and it has also been developed in recent years for the assessment of crack widths. In this technique, the sample is excited by a modulated or pulsed laser spot and the camera records the evolution of the surface temperature distribution, with both the sample and the laser at rest. In this work, we apply laser spot thermography to samples that move at constant velocity, with two main goals: evaluating the thermal diffusivity of the material and assessing the width of infinite vertical cracks on in-line industrial production chains.

First, we have calculated the evolution of the surface temperature distribution when a focused laser spot excites the surface of a material that moves at constant velocity. By analyzing the expressions of surface temperature in logarithmic scale, we present a simple method to measure the in-plane thermal diffusivity in the directions parallel and perpendicular to the motion. In order to check the validity of the methods, we present experimental data on moving reference materials. The retrieved thermal diffusivities are in excellent agreement with literature values.

In the second part, we tackle the assessment of the width of infinite vertical cracks of moving samples. We have calculated the evolution of the surface temperature distribution when the cracked specimen is excited by a cw laser spot. We show that, by fitting the temperature profile perpendicular to the crack, through the center of the laser spot it is possible to measure the thermal resistance (i.e., the width) of the crack. We present experimental data on samples containing artificial calibrated vertical cracks and we discuss on the validity range and limits of the method.

\section{In-plane thermal diffusivity measurements on moving samples}

In order to measure the thermal diffusivity, we have calculated the evolution of the surface temperature distribution of a material that moves at constant velocity $v$ along the $O X$ axis, while it is illuminated by a cw laser spot. The calculation was performed starting from the time domain solution of the static problem (i.e., when a sample at rest is illuminated by a brief light pulse) [1] and performing the convolution product to obtain the temperature when a cw laser spot scans the sample surface in the direction of the $O X$ axis. Then, a simple Galilean transformation of coordinates provides the temperature when the laser stays at rest and the sample moves at constant velocity $v$ in the opposite direction. We have obtained the temperature for different types of samples: opaque or semi-transparent to the incident radiation, thick or thin, and for anisotropic samples with the principal directions coinciding with the direction of motion and the perpendicular.

By analysing the temperature distribution when the steady state is reached, we have found that the plot of the natural logarithm of the temperature multiplied by the transverse (OY) lateral distance to the laser spot is a straight line (see figure 1a). Similarly, when we plot the same kind of profile along the direction of motion (OX), we obtain a flat plateau for positive $x$ coordinates (positions already heated by the laser) and a straight line ahead the laser spot (see figure 1b). The slopes of these straight lines for the longitudinal $\left(m_{\mathrm{p}}\right)$, and transverse $\left(m_{\perp}\right)$ profiles are related to the corresponding longitudinal $\left(D_{\mathrm{p}}\right)$ and transverse $\left(D_{\perp}\right)$ thermal diffusivities by:

$$
m_{\mathrm{p}}=\frac{v}{D_{\mathrm{p}}} \quad m_{\perp}= \pm \frac{v}{2 D_{\perp}}
$$
$\operatorname{Ln}\left(T y^{0.5}\right)$

For thermally thin or semi-transparent materials the same kind of curves are obtained when plotting $\operatorname{Ln}\left(T x^{0.5}\right)$ and

As an example, in figure 1 we present experimental longitudinal and transverse profiles obtained for a thick $\mathrm{Zn}$ slab and two thin plates of $\mathrm{Zn}$ and AISI-304 moving at $6 \mathrm{~cm} / \mathrm{s}$. The thermal diffusivities are obtained from Eqs. (1). We have checked different materials, thick and thin covering a large range of diffusivities and optical absorptions coefficients, 
and in all cases, the retrieved thermal diffusivities are in excellent agreement with literature values, which confirms the validity of the methods.
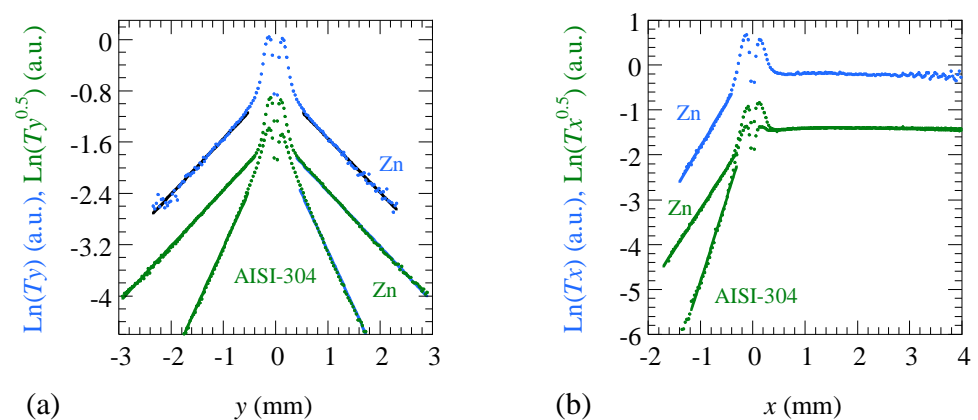

Fig. 1: Experimental (symbols) transverse (a) and longitudinal (b) temperature profiles for a thick slab of Zn and two thin plates of Zn and AISI-304 obtained with samples moving at $6 \mathrm{~cm} / \mathrm{s}$. Solid lines represent the best fit to the model.

\section{Sizing the width of infinite vertical cracks}

Next, we consider a semi-infinite and opaque sample that contains an infinite vertical crack. We have calculated the evolution of the surface temperature distribution when the sample moves ant constant velocity and is illuminated by a cw laser spot, neglecting heat losses by convection and radiation. Following the procedure described in section 2 , we have started from the time domain solution [2] and we have calculated the convolution product, followed by a Galilean transformation of coordinates. According to the sensitivity analysis we have conducted, we propose to fit the surface temperature profile, perpendicular to the crack, through the center of the laser spot, when the distance between the center of the excitation and the crack is approximately equal to the radius of the spot. Furthermore, we propose to use high speed and tightly focused laser spots to size the width of very narrow cracks.

In order to check the procedure, we have taken data on AISI-304 samples containing artificial and calibrated vertical cracks. Figure 2 shows experimental OX profiles (and fittings to the model) obtained on a sample containing a 2.5 $\mu \mathrm{m}$ wide crack, for two speeds: 3.6 and $7.5 \mathrm{~mm} / \mathrm{s}$. As can be seen, the retrieved widths $L$ are in very good agreement with the nominal value. Finally, we discuss on the validity range and the limits of the technique to size cracks.
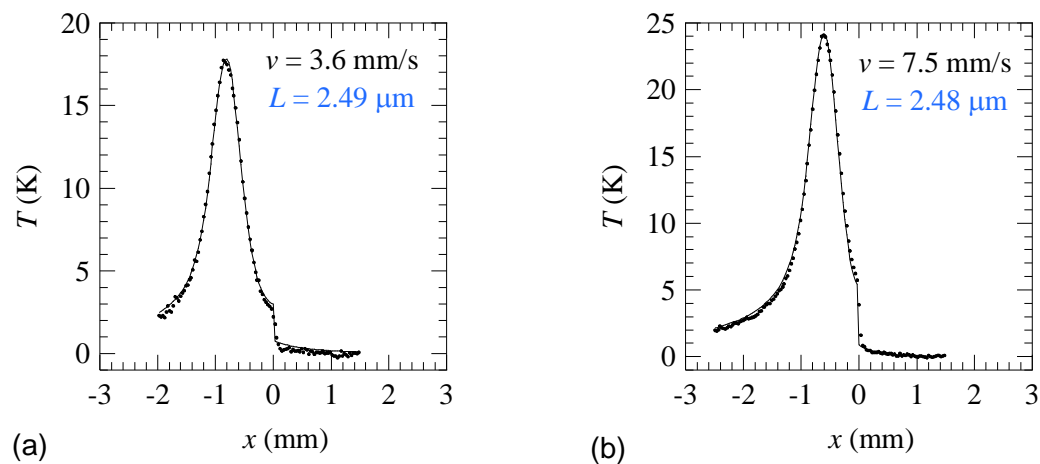

Fig. 2: Experimental OX profiles (symbols), perpendicular to the crack, through the center of the laser spot, for a nominal crack of width $2.5 \mu \mathrm{m}$, at two speeds: (a) $3.6 \mathrm{~mm} / \mathrm{s}$ and (b) $7.5 \mathrm{~mm} / \mathrm{s}$. The continuous line is the fit to the model and the retrieved crack widths are written in blue.

This work has been supported by Ministerio de Economía y Competitividad (DPI2016-77719-R, AEI/FEDER, UE), by Gobierno Vasco (PIBA2018/15) and by Universidad del País Vasco UPV/EHU (GIU16/33).

\section{REFERENCES}

[1] Pech-May NW, Mendioroz A, Salazar A. Simultaneous measurement of the in-plane and in-depth thermal diffusivity of solids using pulsed infrared thermography with focused illumination. NDT\&E Int. 2016;77:28-34.

[2] Pech-May NW, Oleaga A, Mendioroz A, Salazar A. Fast characterization of the width of vertical cracks using pulsed laser spot infrared thermography. J. Nondestruct. Eval. 2016;35:22(10 pp). 